UCB-PTH-05/21

LBNL-58216

hep-th/0508238

\title{
Investigating the Stability of a Nonsupersymmetric Landscape
}

\author{
Yoon Pyo Hong ${ }^{1}$ and Indrajit Mitra ${ }^{1,2}$ \\ ${ }^{1}$ Berkeley Center for Theoretical Physics and Department of Physics \\ University of California, Berkeley, CA 94720-7300 \\ ${ }^{2}$ Theoretical Physics Group, Lawrence Berkeley National Laboratory \\ Berkeley, CA 94720-8162
}

\begin{abstract}
We investigate the classical stability of non-supersymmetric Freund-Rubin compactifications of Type IIB string theory on a product of three-dimensional Einstein spaces $A_{3} \times B_{3}$ with both NS-NS and R-R three-form fluxes turned on through $A_{3}$ and $B_{3}$, and a zero axion. This results in a three parameter family of $A d S_{4}$ vacua, with localized sources such as anti-three-branes or orientifold planes required to cancel the R-R four-form tadpole. We scan the entire space of such solutions for perturbative stability and find that anti-three-branes are unstable to a Jeans-like instability. For orientifold compactifications, we derive a precise criterion which the three-dimensional Einstein spaces have to satisfy in order to be stable.
\end{abstract}

August 2005 


\section{Introduction}

String theory compactified down to four dimensions has a richness of vacua. Studies of these vacua in the context of different string theories are by now a well-developed industry (see e.g. 11, 2, 3, 4, 15, 6, 17, 8, 9, 10, 11, 12, 13, 14, 15, 16]). Counting the number of these vacua has been the focus of many investigations - the considerations of Bousso and Polchinski [17], followed up by the more sophisticated and detailed investigations for vacua preserving $\mathcal{N}=1$ supersymmetry by Douglas and collaborators [18, 19, 20]. This plethora of vacua leads to an embarrassment of richness in making contact with four-dimensional physics. It is therefore interesting to ask how many vacua in this landscape 21] remain perturbatively stable once supersymmetry is broken. In this paper, we examine a class of compactifications of Type IIB string theory which have non-supersymmetric four-dimensional AdS vacua, and perform a stability analysis. (For somewhat related work, see [22. For a study of the stability of compactifications with de Sitter vacua, see e.g. [23, 24.)

Ideally, one would like to start with a model with a large number of supersymmetric vacua, break supersymmetry, and then perform a counting of the number of stable vacua. This is not what we do. Instead, we consider a set-up which is nonsupersymmetric from the start. What we have in mind is a Freund-Rubin compactification [25] of Type IIB on a six-dimensional space $X_{6}$ which is a direct product of three-dimensional positively curved Einstein spaces $A_{3} \times B_{3}$, with 3-form fluxes threading both $A_{3}$ and $B_{3}$. In these solutions the radii of curvature of $A_{3}$ and $B_{3}$ increase with 3-form fluxes. We consider large fluxes where classical supergravity can be trusted. In the absence of supersymmetry, the small radius limit will receive large corrections. ${ }^{1}$ To our knowledge, this class of solutions appeared first in [26]. In this paper we examine the perturbative stability of these solutions.

There are at least three motivations to be interested in the study of the stability of such compactifications:

- These are the simplest compactifications of IIB string theory with non-trivial three-cycles through which one can thread $H_{3}$ and $F_{3}$ flux. It is therefore very interesting to ask how many of the non-supersymmetric vacua are classically stable.

- The flux compactifications under study here arise as near-horizon limits of stacks of NS5 and D5 branes wrapping $A_{3}$ and $B_{3}$. They are therefore similar to the

\footnotetext{
${ }^{1}$ Even in the large radius limit one could still worry that close to the orientifold planes (which we introduce shortly) there are significant corrections to the geometry. Moreover, our very geometric approach to objects that are intrinsically non-geometric might not seem rigorous. We shall gloss over both of these subtleties by hoping that at low energies, our analysis would be nearly correct.
} 
KKLT and KKLMMT models [1, 27] which also have 5-branes wrapping threecycles in a Calabi-Yau. As such, the set-up considered here could be viewed as a toy-model of the more sophisticated Calabi-Yau constructions. ${ }^{2}$ This toymodel might provide some mileage in constructing putative three-dimensional field theory duals, especially since the brane configurations are known here.

- Finally, since the issue of stability of product space compactifications with fluxes threading the individual parts of the product has (to our knowledge) not been carried out (see [28] however for a closely related set-up), the exercise is interesting by itself in getting an idea of when such compactifications are unstable.

When one desires a 4-d AdS vacuum in some theory such as IIB, one still has to choose a particular 6-d compact space, $X_{6}$. In order to be able to turn on 3-form fluxes, one requires that $X_{6}$ have some number of three-cycles. The simplest choice is then a direct product of three-manifolds. Once one has made a choice of $X_{6}$, each of the four dimensional anti-de Sitter vacua are differentiated by the amounts of 3form flux turned on through each three-cycle. In general these would be four integers $\left(n_{1}, n_{2}, q_{1}, q_{2}\right)$, with $n_{1}, n_{2}$ specifying the amount of NS-NS flux through $A_{3}$ and $B_{3}$, respectively, and $q_{1}, q_{2}$ the R-R flux. However, for simplicity, we shall choose an ansatz with no axion turned on. This yields a constraint relating the four integers. There is also a $C_{4}$ tadpole constraint, which is Gauss's law for 3-brane (or $H_{3} \wedge F_{3}$ ) charge in the compact space. A non-zero $H_{3} \wedge F_{3}$ necessitates the introduction of localized objects which carry 3-brane charge. The various options in IIB string theory are D3-branes and O3-planes (or $\overline{\mathrm{D} 3}$ and $\overline{\mathrm{O} 3}$, depending on the relative orientation of $H_{3}$ and $F_{3}$ ), which are point-like in $X_{6}$, or $p$-dimensional branes wrapped on $(p-3)$-cycles in $X_{6}$. For simplicity we consider only the first two options. We find that if we denote the number of localized objects needed to cancel the $C_{4}$ tadpole by $t$, we have a three parameter family of $A d S_{4}$ vacua which are conveniently labelled by $t, \alpha=n_{1} / n_{2}$, and $g_{s}=e^{\phi}$, whose background value we shall see is $\alpha n_{2} / q_{2}$. The entropy of these solutions (which is related to the number of AdS vacua) depends on fluxes, and the relationship can be computed from the value of the four-dimensional cosmological constant using the standard argument of Witten and Susskind [29]. The result is that the entropy is proportional to $t^{2}$. (Recall that for supersymmetric vacua constrained to obey the $C_{4}$ tadpole condition, the entropy scales as $t^{b_{3}}$, where $b_{3}$ is the third Betti number of the compact space. Our ansatz is chosen so that the 3 -form fluxes do not simultaneously have legs on both $A_{3}$ and $B_{3}$, so that $b_{3}$ is effectively 2.)

Let us now examine each of the two simple ways to cancel the $C_{4}$ tadpole. One

\footnotetext{
${ }^{2}$ Recall that in the de Sitter compactifications mentioned above, the first step is to obtain an AdS vacuum. One then adds anti-three-branes which more than cancel the negative cosmological constant to obtain a meta-stable de Sitter vacuum.
} 
solution, which was considered in [26], is to use $\overline{\mathrm{D} 3}$-branes. This nice example, however, has an interesting instability. We know that in flat space, the gravitational attraction of the anti-branes is exactly cancelled by the electrical repulsion due to the $\mathrm{R}-\mathrm{R}$ 5form field strength. In the set-up here however, there is in fact a net attractive force between the anti-branes resulting from the polarization of the flux in between [30]. The background $H_{3} \wedge F_{3}$ can be thought of as an induced D3-brane charge. This charge screens the form-field repulsion, but since the graviational force is unchanged, there is a resultant attractive force. The analysis of [30] indicates that this process of clumping of the anti-branes is very quick.

The other option, using orientifold planes, is free of this instability, since by definition they cannot move. Therefore, we perform a stability analysis with O3-planes. We find, surprisingly, that the mass spectrum is in fact independent of both $g_{s}$ and $\alpha$, but depends only on the orientifold action. The orientifold action is $\Omega R(-1)^{F_{L}}$ [31, 32, 33, where $\Omega$ is the world-sheet parity, $R$ is the $\mathbf{Z}_{2}$ action on $X_{6}$, and $F_{L}$ is the spacetime fermion number of the left-movers. We shall be looking at an ansatz with constant three-form fluxes $H_{3}$ and $F_{3}$ which are even under the $\mathbf{Z}_{2}$. The other fields such as the axion, dilaton, and the metric are also even. In this case, we have the result that most choices of $A_{3}$ and $B_{3}$ will lead to instabilities! The precise criterion we find is that there is an instability if there is an eigenfunction of the scalar Laplacian operator on $A_{3}$ (or $B_{3}$ ) which is even under the $\mathbf{Z}_{2}$ action and has eigenvalue $3 \leq-R^{2} \square<22.1$, where $R$ is the radius of curvature of $A_{3}$ (and $B_{3}$ ). The mode is tachyonic in the sense that its mass is below the Breitenlohner-Freedman bound [34]. Indeed, we give an explicit example where the compact space is chosen to be $S^{3} \times S^{3}$, which turns out to be unstable. It would be interesting to have an explicit example of $A_{3}$ and $B_{3}$ with no eigenvalue of the Laplacian in the above range, or in which the orientifold action projects out the unstable mode.

In this paper we shall carry out the stability analysis along the lines of [35, 36, 37. 38. In this approach, we shall be able to compute the spectrum of the entire KK tower of fluctuations about the background. Often the approach is to perform a zeromode (or s-wave) reduction of the higher dimensional theory down to four dimensions. The advantage of the latter method is that one clearly sees the various contributions to the four-dimensional effective potential for the different fields such as the size of the compact space, dilaton, etc. Some of the contributions, like fluxes, contribute positively, while others, such as positive curvature of the compact space and negative tension objects, have negative contributions. The various 'forces' stabilizing the moduli come at different orders in the string perturbation theory: for example, the 4-d vacuum energy from compactifying on a compact space and the energy from NS-NS fluxes are at string tree-level, while examples of contributions at one-loop include R-R fluxes and gaugino condensates [39. In fact, sometimes the contribution could be even non- 
perturbative, for instance from Euclidean branes as in [1, 27]. A nice review of these various types of contributions are listed in the lecture notes [26]. The term "moduli stabilization" is used to indicate solutions in which the potential has at least one minimum with finite non-zero values of every field. From the ten-dimensional point of view, this simply means that there are non-trivial solutions to the equations of motion.

Although this s-wave effective potential is an excellent guide in computing the background, we should remind ourselves that it can be quite misleading in informing us of the stability of the background. In other words, the fact that the background sits at the minimum of the effective potential is not a guarantee for stability. In fact, there could be a tachyonic instability in a low-lying KK fluctuation which is not an s-wave. ${ }^{3}$ It is for this reason that we inspect the low-lying tower of states for this class of compactifications. It is a highly non-trivial matter to sieve through all the modes, and in practice one uses some judgement to pick out modes which are usually least massive to determine stability. Keeping this in perspective, we shall use the lessons learned in 38, 40] (see also [28]) about product-space compactifications.

The most obvious source of instability for product spaces $X_{q}=A_{n} \times B_{q-n}$ is an instability in which one part, say $A_{n}$, expands, while the other part $B_{q-n}$ shrinks, preserving the total volume locally. In fact [38, 40] showed that $(p+q)$-dimensional Einstein-Maxwell theories with a $q$-form flux, when compactified on a $q$-dimensional positively curved Einstein space, had only precisely this instability. But results of that analysis do not apply to the example that we have in mind because here we do not have a 6-form flux threading the $A_{3} \times B_{3}$. Instead, we have both R-R and NS-NS 3-form fluxes threading each three-cycle. The presence of the 3 -form fluxes would lead one to expect that this mode would be stabilized. More generally, the class of modes that we consider is the coupled scalar sector, consisting of the traces of the metric fluctuations on $\mathrm{AdS}$, on $A_{3}$ and $B_{3}$, the fluctuations of the dilaton and axion, and the fluctuation of the R-R and NS-NS 3-form fields. We compute the masses of these modes.

This paper is organized as follows. In Section 2 we explain the set-up and determine the background from a ten-dimensional perspective. ${ }^{4}$ We point out the two possible choices of cancelling the $C_{4}$ tadpole, by adding mobile $\overline{\mathrm{D} 3}$-branes or by adding O3-planes, and then discuss possible instabilities. Section 3 discusses the growth of the number of $A d S_{4}$ vacua with 3 -form fluxes. Finally, Section 4 , which is the most important section of the paper, determines the set of vacua which are stable.

\footnotetext{
${ }^{3}$ See for instance the result in 38 in which the lowest mass mode in the coupled scalar sector was a mode whose "orbital angular momentum" was $\frac{3}{4}(q-1)^{2}$, where $q$ is the dimension of the compact manifold.

${ }^{4}$ For a four-dimensional treatment of the background, see [26].
} 


\section{$2 \mathrm{AdS}_{4}$ Vacua in IIB}

The bosonic part of the IIB action in Einstein frame is [4]

$$
\begin{aligned}
S_{\mathrm{IIB}}=\frac{1}{2 \kappa_{10}^{2}} \int & d^{10} x \sqrt{-g}\left[R-\frac{1}{2}(\partial \phi)^{2}-\frac{1}{2} e^{2 \phi}\left(\partial C_{0}\right)^{2}-\frac{1}{2}\left(C_{0}^{2} e^{\phi}+e^{-\phi}\right)\left|H_{3}\right|^{2}\right. \\
& \left.+C_{0} e^{\phi} H_{3} \cdot F_{3}-\frac{1}{2} e^{\phi}\left|F_{3}\right|^{2}-\frac{1}{4}\left|\tilde{F}_{5}\right|^{2}\right]+\frac{1}{4 \kappa_{10}^{2}} \int C_{4} \wedge F_{3} \wedge H_{3},
\end{aligned}
$$

where $\tilde{F}_{5}$ is defined as

$$
\tilde{F}_{5}=d C_{4}-\frac{1}{2} C_{2} \wedge H_{3}+\frac{1}{2} B_{2} \wedge F_{3}
$$

The equations of motion obtained by varying this action are

$$
\begin{aligned}
R_{M N} & =\frac{1}{2} \partial_{M} \phi \partial_{N} \phi+\frac{1}{2} e^{2 \phi} \partial_{M} C_{0} \partial_{N} C_{0}+\left(C_{0}^{2} e^{\phi}+e^{-\phi}\right)\left[\frac{1}{2 \cdot 2 !} H_{M P_{1} P_{2}} H_{N} P_{1} P_{2}-\frac{1}{8} g_{M N}\left|H_{3}\right|^{2}\right] \\
& -C_{0} e^{\phi}\left[\frac{1}{2 \cdot 2 !} H_{M P_{1} P_{2}} F_{N} P_{1} P_{2}+\frac{1}{2 \cdot 2 !} F_{M P_{1} P_{2}} H_{N} P_{1} P_{2}-\frac{1}{4} g_{M N} H_{3} \cdot F_{3}\right] \\
& +e^{\phi}\left[\frac{1}{2 \cdot 2 !} F_{M P_{1} P_{2}} F_{N} P_{1} P_{2}-\frac{1}{8} g_{M N}\left|F_{3}\right|^{2}\right]+\frac{1}{4 \cdot 4 !} \tilde{F}_{M P_{1} P_{2} P_{3} P_{4}} \tilde{F}_{N} P_{1} P_{2} P_{3} P_{4}, \\
0 & =\square \phi-e^{2 \phi}\left(\partial C_{0}\right)^{2}-\frac{1}{2} e^{\phi}\left|F_{3}\right|^{2}+C_{0} e^{\phi} H_{3} \cdot F_{3}-\frac{1}{2}\left(C_{0}^{2} e^{\phi}-e^{-\phi}\right)\left|H_{3}\right|^{2}, \\
0 & =\square C_{0}+2 \partial_{M} \phi \partial^{M} C_{0}-C_{0} e^{-\phi}\left|H_{3}\right|^{2}+e^{-\phi} H_{3} \cdot F_{3}, \\
0 & =d *\left(C_{0}^{2} e^{\phi}+e^{-\phi}\right) H_{3}-d *\left(C_{0} e^{\phi} F_{3}\right)-F_{3} \wedge * \tilde{F}_{5}, \\
0 & =d *\left(e^{\phi} F_{3}\right)-d *\left(C_{0} e^{\phi} H_{3}\right)+H_{3} \wedge * \tilde{F}_{5}, \\
0 & =d * \tilde{F}_{5}-H_{3} \wedge F_{3}, \quad \tilde{F}_{5}=* \tilde{F}_{5} .
\end{aligned}
$$

We shall consider Freund-Rubin compactifications of the form

$$
\begin{aligned}
d s^{2} & =d s_{\mathrm{AdS}_{4}}^{2}+d s_{A_{3}}^{2}+d s_{B_{3}}^{2}, \\
H_{3} & =N_{1} \operatorname{vol}_{A_{3}}+N_{2} \operatorname{vol}_{B_{3}}, \quad F_{3}=Q_{1} \operatorname{vol}_{A_{3}}+Q_{2} \operatorname{vol}_{B_{3}},
\end{aligned}
$$

where $\mathrm{vol}_{A_{3}}$ is the volume form of $A_{3}$ and similarly for $B_{3}$. We use upper-case Roman letters $M, N, \ldots$, for indices on the full 10-dimensional spacetime, Greek letters $\mu, \nu, \ldots$, for indices on AdS, lower-case Roman letters $a, b, \ldots$, for indices on $A_{3}$, and $i, j, \ldots$, for indices on $B_{3}$. Since we are dealing with Einstein spaces, the Ricci tensor is related to the metric tensor as follows:

$$
R_{\mu \nu}=-\frac{3}{L^{2}} g_{\mu \nu}, \quad R_{a b}=\frac{2}{R_{1}^{2}} g_{a b}, \quad R_{i j}=\frac{2}{R_{2}^{2}} g_{i j},
$$


where $L, R_{1}$, and $R_{2}$ are radii of curvature of $\mathrm{AdS}_{4}, A_{3}$, and $B_{3}$, respectively.

The IIB action has an $\mathrm{SL}(2, \mathbb{Z})$ symmetry of the axion-dilaton. We focus on the simplest ansatz and choose a zero axion and constant dilaton. The condition $C_{0}=0$ implies

$$
N_{1} Q_{1}+N_{2} Q_{2}=0 .
$$

A convenient parametrization of the four fluxes satisfying the constraint (6) is

$$
N_{1}=\alpha N, \quad N_{2}=N, \quad Q_{1}=-\frac{1}{\alpha} Q, \quad Q_{2}=Q,
$$

where we restrict $\alpha, N$, and $Q$ to taking on positive values. Furthermore, requiring a constant dilaton results in

$$
e^{\phi}=\frac{\alpha N}{Q} .
$$

Integrating the $\tilde{F}_{5}$ equation of motion over the compact space gives us a non-trivial Gauss's law constraint on the compact space $X_{6}$ :

$$
0=\int_{X_{6}} H_{3} \wedge F_{3}=\left(N_{1} Q_{2}-N_{2} Q_{1}\right) \cdot\left(\text { volume of } X_{6}\right),
$$

which is clearly incompatible with (6). To make the net three-brane charge vanish we introduce local objects which carry this charge such as D3 (and $\overline{\mathrm{D} 3}$ ) branes and O3 (and $\overline{\mathrm{O} 3}$ ) planes as in [42. These sources are localized on the compact space and extend along AdS. The full action including the localized sources is now

$$
S=S_{\text {IIB }}+S_{\text {local }} \quad \text { where } \quad S_{\text {local }}=-T_{3} \int d^{4} x \sqrt{-g}+\mu_{3} \int C_{4} .
$$

$T_{3}$ and $\mu_{3}$ are the three-brane tension and charge, respectively. This modifies the $\tilde{F}_{5}$ equation of motion (3) to

$$
d * \tilde{F}_{5}=H_{3} \wedge F_{3}+2 \kappa_{10}^{2} T_{3}\left(\rho_{D}-\frac{1}{4} \rho_{O}\right),
$$

where $\rho_{D}$ and $\rho_{O}$ are the number densities of D3-branes and O3-planes on the compact space, respectively. The corresponding global constraint is now [26]

$$
\frac{1}{2(2 \pi)^{4}\left(\alpha^{\prime}\right)^{2}} \int_{X_{6}} H_{3} \wedge F_{3}=N_{\overline{D 3}}-N_{D 3}+\frac{1}{4}\left(N_{O 3}-N_{\overline{O 3}}\right) .
$$

Note that we have put an extra factor of 2 on the left-hand side, anticipating $N_{O 3} \neq 0$. This arises in the presence of O3-planes because in (11) we are integrating over the full covering space instead of the $\mathbf{Z}_{2}$ quotient. 
Let us recall that the 3-form fluxes are quantized; that is,

$$
\begin{aligned}
n_{1} & =\frac{1}{(2 \pi)^{2} \alpha^{\prime}} \int_{A_{3}} H_{3}, & n_{2} & =\frac{1}{(2 \pi)^{2} \alpha^{\prime}} \int_{B_{3}} H_{3}, \\
q_{1} & =\frac{1}{(2 \pi)^{2} \alpha^{\prime}} \int_{A_{3}} F_{3}, & q_{2} & =\frac{1}{(2 \pi)^{2} \alpha^{\prime}} \int_{B_{3}} F_{3},
\end{aligned}
$$

are all integers. In the presence of orientifold planes, because of the $\mathbf{Z}_{2}$ action on $A_{3}$ and $B_{3}$, we restrict the integers $n_{1}, n_{2}, q_{1}, q_{2}$ to even values. ${ }^{5}$ Furthermore, from Eq. (17) we obtain $\alpha=n_{1} / n_{2}=\left|q_{2} / q_{1}\right|$, which prevents $\alpha$ from varying continuously and restricts it to taking only rational values. In fact, there is a further restriction that $\alpha$ has to satisfy, which comes from the tadpole constraint. To see this, let us combine (12) with (11), which allows us to express the number of localized objects in terms of three-form flux quanta:

$$
\frac{1}{2}\left(n_{1} q_{2}-n_{2} q_{1}\right)=\frac{1}{2}\left(\alpha+\frac{1}{\alpha}\right) n q=N_{\overline{D 3}}-N_{D 3}+\frac{1}{4}\left(N_{O 3}-N_{\overline{O 3}}\right)
$$

where $n=n_{2}$ and $q=q_{2}$. Since we restrict ourselves to the cases with positive $\alpha, n$, and $q$, we shall now consider the following two cases: (a) only $N_{\bar{D} 3} \neq 0$, and (b) only $N_{O 3} \neq 0$.

In the first option, one puts mobile anti-D3 branes with their world-volumes along $A d S_{4}$ and each localized as a point on $X_{6}$. Let us examine what happens to the system if we start with a uniform distribution of branes on $X_{6}$. To determine whether this system is stable, it suffices to consider the interaction of two anti-branes. In flat space, a pair of anti-D3 branes would be mutually BPS - their attractive gravitational force exactly cancels the repulsive R-R force. When $A_{3}$ and $B_{3}$ are three-spheres, this is also true to leading order. But in our set-up there is more to the picture. There is also a net $H_{3} \wedge F_{3}$ flux threading the three-spheres. From (11) it is clear that the flux acts as a cloud of positive 3-brane charge. This screens the negative charge on each anti-brane and therefore, the gravitational attraction is now stronger than the R-R repulsive force. This interesting instability due to flux polarization was encountered in [30. To sum up, a Jeans instability sets in and the anti-branes clump on the compact space, possibly followed by the interesting dynamics studied in great detail in [30]. It would be interesting to investigate whether supersymmetry is restored in the end, but we shall not do so here.

The second option, that of introducing O3-planes, is what we shall look into in some detail in this paper. Like the anti-D3 branes above, these orientifold planes also lie completely along AdS. It is important to realize that the number of such planes that

\footnotetext{
${ }^{5}$ See however, 32, 33 for how this restriction could be lifted by considering O3-planes with discrete fluxes and with $+1 / 4$ D3 charge.
} 
can be introduced is not arbitrary, but depends on the symmetries of the compact space. More specifically, one looks for fixed points of the action of a subgroup $\Gamma$ of the isometry group of $X_{6}$. For the example in which both $A_{3}$ and $B_{3}$ are simply threespheres, $\Gamma$ is a subgroup of $\mathrm{SO}(4) \times \mathrm{SO}(4)$. The number of such orientifold planes which are allowed are determined by the choice of $X_{6}$ and $\Gamma$. We shall denote the number of such O3-planes by $t$ in this paper, and view (13) as a constraint on the fluxes. Combining this with Eqs. (7) and (8), we can now parameterize the space of vacua with three numbers $\left(g_{s}, \alpha, t\right)$, where $g_{s}=\alpha N / Q$ is determined from the background value of the dilaton, $\alpha$ can be thought of as an asymmetry parameter measuring the ratio of a given kind of flux on $A_{3}$ to that along $B_{3}$, and finally, $t=N_{O 3}$ is just the number of O3-planes.

The introduction of localized objects changes the stress-energy tensor $T_{M N}$ and so the background geometry gets corrected. To compute the radii of curvature $L, R_{1}$, and $R_{2}$, let us write Einstein's equation as

$$
R_{M N}=\kappa_{10}^{2}\left(T_{M N}-\frac{1}{8} g_{M N} T_{P}^{P}\right) .
$$

Let us denote the collective coordinates on $A_{3}$ by $y$ and those on $B_{3}$ by $z$, and let $y_{k}$ and $z_{k}$ be the coordinates of the $k$-th orientifold plane on $X_{6}$. Then their contribution to the stress-energy tensor is given by

$$
T_{\mu \nu}^{\mathrm{loc}}=\frac{T_{3}}{4} g_{\mu \nu} \sum_{k} \delta^{3}\left(y-y_{k}\right) \delta^{3}\left(z-z_{k}\right), \quad T_{a b}^{\mathrm{loc}}=T_{i j}^{\mathrm{loc}}=0,
$$

where $-T_{3} / 4$ is the tension of an O3-plane (so that $2 \kappa_{10}^{2} T_{3}=(2 \pi)^{4} \alpha^{\prime 2}$ ). Together with the contribution from the supergravity fields in (3), Einstein's equations (14) now reads

$$
R_{\mu \nu}=-\frac{3}{L^{2}} g_{\mu \nu}=-\frac{1}{4} g_{\mu \nu}\left(\alpha+\frac{1}{\alpha}\right) N Q+\frac{1}{8} \kappa_{10}^{2} T_{3} g_{\mu \nu} \sum_{k} \delta^{3}\left(y-y_{k}\right) \delta^{3}\left(z-z^{k}\right)
$$

along the AdS directions. Taking traces of $R_{M N}$ first along $A_{3}$ and then along $B_{3}$ we also get

$$
\begin{aligned}
& R_{a}^{a}=\frac{6}{R_{1}^{2}}=\frac{3}{4}\left(\alpha+\frac{1}{\alpha}\right) N Q-\frac{3}{8} \kappa_{10}^{2} T_{3} \sum_{k} \delta^{3}\left(y-y_{k}\right) \delta^{3}\left(z-z_{k}\right), \\
& R_{i}^{i}=\frac{6}{R_{2}^{2}}=\frac{3}{4}\left(\alpha+\frac{1}{\alpha}\right) N Q-\frac{3}{8} \kappa_{10}^{2} T_{3} \sum_{k} \delta^{3}\left(y-y_{k}\right) \delta^{3}\left(z-z_{k}\right) .
\end{aligned}
$$

From now on, we denote the (common) radius of curvature of $A_{3}$ and $B_{3}$ by $R$.

In order for the supergravity approximation to hold, the radius of curvature of our background must be large compared to string scale. Therefore, we take the limit 
$R / \sqrt{\alpha^{\prime}} \gg 1$ with dimensionless combinations $R N$ and $R Q$ fixed. From the quantization condition (12), we see that this is the limit of large number of flux quanta on $A_{3}$ and $B_{3}$, and hence we need a large number of O3-planes. Now, the number of O3-planes per unit volume of $X_{6}$ scales like $n q / R^{6} \sim 1 / R^{2}\left(\alpha^{\prime}\right)^{2}$, so the distance between adjacent O3's will roughly be $\sim\left(R \alpha^{\prime}\right)^{1 / 3}$. Therefore, in the low energy limit where we are only interested in the length scale, say $\lambda>R^{2 / 3}\left(\alpha^{\prime}\right)^{1 / 6}$, we will not be able to see individual O3-planes. Rather, in this low energy limit, we can replace their delta function distribution in (15) by a uniform distribution [42, 43, 44, with density

$$
\rho=\frac{2}{(2 \pi)^{4} \alpha^{2}}\left(\alpha+\frac{1}{\alpha}\right) N Q .
$$

Then $\tilde{F}_{5}=0$ is a solution of (10) in this limit, and Einstein's equations (16) and (17) determine the radii of $\mathrm{AdS}$ and those of $A_{3}$ and $B_{3}$ in terms of 3-form fluxes. Our background is:

$$
\frac{3}{L^{2}}=\frac{2}{R_{1}^{2}}=\frac{2}{R_{2}^{2}}=\frac{1}{8}\left(\alpha+\frac{1}{\alpha}\right) N Q, \quad e^{\phi}=\frac{\alpha N}{Q} .
$$

\section{Number of Vacua and the 4D Viewpoint}

The first thing one wants to know about the holographic dual of a string theory in AdS is the number of degrees of freedom of the field theory. For instance, for string theory on $A d S_{5} \times S^{5}$, which is dual to $\mathcal{N}=4$ theory with gauge group $\mathrm{SU}(N)$, has $N^{2}$ degrees of freedom. It turns out that AdS/CFT allows one to predict the degrees of freedom at strong coupling by the Susskind-Witten arguments [29]. On the gravity side one computes the cosmological constant of the reduced theory (in the $A d S_{5} \times S^{5}$ example, this is the 5 - $\mathrm{d}$ cosmological constant); the entropy is then inversely proportional to this. For 4-d theories the entropy is

$$
S \sim \frac{1}{l_{4}^{2} \Lambda_{4}^{E}}
$$

where $l_{4}$ is the 4 -d Planck length and $\Lambda_{4}^{E}$ is the 4-d cosmological constant (measured in the Einstein frame). Let us compute the entropy for our $A d S_{4}$ vacua which result from $A_{3} \times B_{3}$ compactifications that we have considered in this paper.

We shall compute $\Lambda_{4}^{E}$ from the reduced 4-d effective potential that we mentioned in the Introduction. This will tell us about the shape of the s-wave effective potential, reminding us of the various positive and negative contributions to the vacuum energy. In this section we follow the treatment in [26]. 
The string frame cosmological term is obtained by integrating various terms in the string-frame action over the compact space $X_{6}$. One gets

$$
\Lambda_{4}^{s} \sim e^{-2 \phi}\left(-\frac{R_{1}^{3} R_{2}^{3}}{R_{1}^{2}}-\frac{R_{1}^{3} R_{2}^{3}}{R_{2}^{2}}+\frac{n_{1}^{2} R_{2}^{3}}{R_{1}^{3}}+\frac{n_{2}^{2} R_{1}^{3}}{R_{2}^{3}}\right)+\left(\frac{q_{1}^{2} R_{2}^{3}}{R_{1}^{3}}+\frac{q_{2}^{2} R_{1}^{3}}{R_{2}^{3}}\right)-t e^{-\phi}
$$

where we have separated the contributions due to the NS-NS fields, the R-R fields, and finally the O3-planes. Everything is measured in string units. The metric in the string frame is related to that in the Einstein frame by

$$
g_{M N}^{E}=e^{-\phi / 2} g_{M N}^{s} .
$$

From this, one can show that the 4-d cosmological constant in the Einstein frame and string frame are related by

$$
l_{4}^{2} \Lambda_{4}^{E}=\frac{e^{4 \phi}}{V_{X}^{2}} \Lambda_{4}^{s}
$$

where $V_{X}$ is the volume of the compact space. To get just the scalings, we assume $n_{1}=n_{2}=n$ and $q_{1}=q_{2}=q$ in this section (in the notation of the rest of the paper, this is the same as setting $\alpha=1$ ). Minimizing the energy (21) with respect to $R_{1}$ and $R_{2}$ immediately yields $R_{1}=R_{2} \sim \sqrt{n}$, while minimizing with respect to $\phi$ yields $e^{\phi} \sim n / q$. These are exactly the same relations as we obtained in (19) by solving the ten-dimensional equations of motion. ${ }^{6}$ Inserting these in (21), we find that each term scales the same, and that

$$
\frac{1}{S} \sim\left(\frac{n}{q}\right)^{4} \frac{1}{n^{3} n^{3}}\left[\frac{n^{3 / 2} n^{3 / 2}}{n} \frac{q^{2}}{n^{2}}+\cdots\right] \sim \frac{1}{(q n)^{2}} \sim \frac{1}{t^{2}} .
$$

So we see that the entropy of these solutions depends on the number of orientifold planes (or $\overline{\mathrm{D} 3}$-branes for that matter) as:

$$
S \sim t^{2}
$$

It is interesting to see how this compares with the number of flux vacua obtained by the Bousso-Polchinski arguments [17. In [26] for instance, this was heuristically sketched out and it was found that

$$
N_{\text {Vacua }} \sim \frac{t^{b_{3}}}{b_{3} !}
$$

where $b_{3}$ is the third Betti number. For $X_{6}=S^{3} \times S^{3}$, we find that (25) and (26) agree. For other compact spaces $b_{3}$ may be different, but the fact that we do not allow the 3-form fluxes to simultaneously have legs on both $A_{3}$ and $B_{3}$ is responsible for the dependence above. It is very interesting to ask if one can account for the entropy in (25) by moving into the Coulomb branch and counting string junctions as in [45].

\footnotetext{
${ }^{6}$ To show this recall that $n \sim N R_{E}^{3}$, and $q \sim Q R_{E}^{3}$. From (22) one has $R_{E}^{2}=e^{-\phi / 2} R_{s}^{2}$.
} 


\section{How Many of These Vacua Are Stable?}

In anti-de Sitter space, the criterion for stability was computed by Breitenlohner and Freedman [34, who showed that in $\mathrm{AdS}_{4}$, for a (normalizable) scalar field to be stable, its mass has to be greater than the so-called BF bound:

$$
m^{2} L^{2} \geq-\frac{9}{4}
$$

In this section, we shall examine perturbative stability of the vacuum solution described in Section 2 against small fluctuations. The particular excitations we shall be interested in are fields which are scalars in the dimensionally reduced theory on $A d S_{4}$. The ten-dimensional origin of these scalars are the trace of the graviton on the $A_{3} \times B_{3}$ (or alternatively, as we shall see, the trace on the metric fluctuations of $A d S_{4}$ ), the dilaton, the axion, and scalar fluctuations of the 3-form fields.

\subsection{Fluctuations}

Let us introduce the following notations for the fluctuation of metric:

$$
\begin{gathered}
\delta g_{\mu \nu}=h_{\mu \nu}=H_{\mu \nu}-\frac{1}{2} g_{\mu \nu}\left(h_{a}^{a}+h_{i}^{i}\right), \\
\delta g_{\mu a}=h_{\mu a}, \quad \delta g_{\mu i}=h_{\mu i}, \quad \delta g_{a b}=h_{a b}, \quad \delta g_{i j}=h_{i j}, \quad \delta g_{a i}=h_{a i} .
\end{gathered}
$$

In Eq. (28), we have defined the standard linearized Weyl shift on $h_{\mu \nu}$. It will be useful to decompose $H_{\mu \nu}, h_{a b}$, and $h_{i j}$ into trace and traceless parts:

$$
H_{\mu \nu}=H_{(\mu \nu)}+\frac{1}{4} g_{\mu \nu} H_{\rho}^{\rho}, \quad h_{a b}=h_{(a b)}+\frac{1}{3} g_{a b} h_{c}^{c}, \quad h_{i j}=h_{(i j)}+\frac{1}{3} g_{i j} h_{k}^{k},
$$

so that $g^{\mu \nu} H_{(\mu \nu)}=g^{a b} h_{(a b)}=g^{i j} h_{(i j)}=0$. We also introduce the fluctuation of the form fields ${ }^{7}$

$$
\delta H_{3} \equiv h_{3}=d b_{2} \quad \delta F_{3} \equiv f_{3}=d c_{2}
$$

and the fluctuations $\delta \phi$ and $\delta C_{0}$ of dilaton and axion fields.

To fix the internal diffeomorphisms and gauge freedom, and to simplify our analysis, we impose the de Donder-type gauge conditions

$$
\nabla^{a} h_{(a b)}=\nabla^{i} h_{(i j)}=0, \quad \nabla^{a} h_{\mu a}=\nabla^{i} h_{\mu i}=\nabla^{a} h_{a i}=\nabla^{i} h_{a i}=0,
$$

on the metric fluctuations and the Lorenz-type conditions

$$
\begin{array}{ll}
\nabla^{a} b_{\mu a}=\nabla^{a} b_{a i}=\nabla^{a} b_{a b}=0, & \nabla^{i} b_{\mu i}=\nabla^{i} b_{a i}=\nabla^{i} b_{i j}=0, \\
\nabla^{a} c_{\mu a}=\nabla^{a} c_{a i}=\nabla^{a} c_{a b}=0, & \nabla^{i} c_{\mu i}=\nabla^{i} c_{a i}=\nabla^{i} c_{i j}=0,
\end{array}
$$

\footnotetext{
${ }^{7}$ Beware that we use the same letter $h$ to denote both the metric and NS-NS 3-form perturbations; they are distinguished by the number of indices they carry.
} 
on the form fluctuations. As we mentioned above, we restrict our analysis to scalar modes in AdS. In the above gauge, we can expand the fluctuations in terms of scalar harmonics of $A_{3}$ and $B_{3}$ as follows:

$$
\begin{aligned}
\delta \phi & =\sum_{I J} \delta \phi^{I J}(x) Y^{I}(y) Z^{J}(z), & \delta C_{0} & =\sum_{I J} \delta C_{0}^{I J}(x) Y^{I}(y) Z^{J}(z), \\
b_{a b} & =\sum_{I J} b_{(1)}^{I J}(x) \epsilon_{a b}{ }^{c} \nabla_{c} Y^{I}(y) Z^{J}(z), & b_{i j} & =\sum_{I J} b_{(2)}^{I J}(x) Y^{I}(y) \epsilon_{i j}{ }^{k} \nabla_{k} Z^{J}(z), \\
c_{a b} & =\sum_{I J} c_{(1)}^{I J}(x) \epsilon_{a b}{ }^{c} \nabla_{c} Y^{I}(y) Z^{J}(z), & c_{i j} & =\sum_{I J} c_{(2)}^{I J}(x) Y^{I}(y) \epsilon_{i j}{ }^{k} \nabla_{k} Z^{J}(z), \\
h_{a}^{a} & =\sum_{I J} \pi_{(1)}^{I J}(x) Y^{I}(y) Z^{J}(z), & h_{i}^{i} & =\sum_{I J} \pi_{(2)}^{I J}(x) Y^{I}(y) Z^{J}(z), \\
H_{\mu}^{\mu} & =\sum_{I J} H^{I J}(x) Y^{I}(y) Z^{J}(z) . & &
\end{aligned}
$$

Here, $Y^{I}$ and $Z^{J}$ are the scalar spherical harmonics on $A_{3}$ and $B_{3}$, respectively. Note that the gauge conditions (33) imply that the two-forms $b_{a b}, b_{i j}, c_{a b}, c_{i j}$ are co-exact on the respective 3-spaces; in (35) and (36), we used the fact that co-exact two-forms on 3-manifolds can be expressed as the Hodge dual of the exterior derivatives of scalars.

\subsection{Fluctuation Equations}

In this section we derive the linearized equations of motion for the fluctuations defined in the previous subsection. We shall follow the analysis of [38] quite closely. Although product spaces were dealt with in that paper, the set-up differed in that there was a 6-form field threading the $S^{3} \times S^{3}$, instead of 3 -form fields like we have here.

Let us consider the dilaton equation first. Expanding the dilaton equation of motion to first order in fluctuations yields

$$
\begin{aligned}
0=\square \delta \phi & -\frac{1}{2}\left(e^{-\phi}\left|H_{3}\right|^{2}+e^{\phi}\left|F_{3}\right|^{2}\right) \delta \phi \\
& +\frac{e^{-\phi}}{2}\left[\frac{2}{3 !} H_{a b c} h^{a b c}+\frac{2}{3 !} H_{i j k} h^{i j k}-\left(N_{1}^{2} h_{a}^{a}+N_{2}^{2} h_{i}^{i}\right)\right] \\
& -\frac{e^{\phi}}{2}\left[\frac{2}{3 !} F_{a b c} f^{a b c}+\frac{2}{3 !} F_{i j k} f^{i j k}-\left(Q_{1}^{2} h_{a}^{a}+Q_{2}^{2} h_{i}^{i}\right)\right] .
\end{aligned}
$$

As introduced in Section 4.1, we write the fluctuations of the field strengths as $h_{3}=d b_{2}$ and $f_{3}=d c_{2}$, where

$$
\begin{aligned}
& b_{2}=\frac{1}{2} b_{a b} d y^{a} \wedge d y^{b}+\frac{1}{2} b_{i j} d z^{i} \wedge d z^{j}, \\
& c_{2}=\frac{1}{2} c_{a b} d y^{a} \wedge d y^{b}+\frac{1}{2} c_{i j} d z^{i} \wedge d z^{j} .
\end{aligned}
$$


Note that we have set the fluctuation modes $b_{\mu \nu}, b_{\mu a}, b_{\mu i}, c_{\mu \nu}, c_{\mu a}, c_{\mu i}$ to zero, because we are interested only in the scalar fluctuations. It can be readily shown that these modes do not enter in the equations of motion for the scalar modes in AdS. Then we can expand each mode in terms of the spherical harmonics as in (35) and (36). After a straightforward algebra, the dilaton fluctuation equation (39) reduces to ${ }^{8}$

$$
\begin{aligned}
& {\left[\left\{\square_{x}+\square_{y}+\square_{z}-\left(\alpha+\frac{1}{\alpha}\right) N Q\right\} \delta \phi^{I J}-\frac{1}{2}\left(\alpha-\frac{1}{\alpha}\right) N Q\left(\pi_{(1)}^{I J}-\pi_{(2)}^{I J}\right)\right.} \\
& \left.\quad+\frac{Q}{\alpha N}\left(N_{1} \square_{y} b_{(1)}^{I J}+N_{2} \square_{z} b_{(2)}^{I J}\right)-\frac{\alpha N}{Q}\left(Q_{1} \square_{y} c_{(1)}^{I J}+Q_{2} \square_{z} c_{(2)}^{I J}\right)\right] Y^{I} Z^{J}=0 .
\end{aligned}
$$

A similar calculation results in the following fluctuation equation for the axion:

$$
\begin{aligned}
& {\left[\left\{\square_{x}+\square_{y}+\square_{z}-\left(\alpha+\frac{1}{\alpha}\right) N Q\right\} \delta C_{0}^{I J}+\frac{Q^{2}}{\alpha}\left(\pi_{(1)}^{I J}-\pi_{(2)}^{I J}\right)\right.} \\
& \left.\quad+\frac{Q}{\alpha N}\left(Q_{1} \square_{y} b_{(1)}^{I J}+Q_{2} \square_{z} b_{(2)}^{I J}+N_{1} \square_{y} c_{(1)}^{I J}+N_{2} \square_{z} c_{(2)}^{I J}\right)\right] Y^{I} Z^{J}=0 .
\end{aligned}
$$

The fluctuation equations for $H_{3}$ and $F_{3}$ are similar, so we shall elaborate the computation for the $H_{3}$ fluctuation and write down only the answer for the $F_{3}$ fluctuation. Using the fact that $C_{0}=0$ in our background, the variation of $H_{3}$ equation of motion reads

$$
d(\delta *)\left(e^{-\phi} H_{3}\right)-d *\left(e^{-\phi} \delta \phi H_{3}\right)+d *\left(e^{-\phi} h_{3}\right)-d *\left(\delta C_{0} e^{\phi} F_{3}\right)=0 .
$$

Here, $\delta *$ in the first term denotes the variation of the Hodge star operator due to the metric fluctuation, which turns out to be

$$
\begin{aligned}
d(\delta *)\left(e^{-\phi} H_{3}\right)=\frac{Q}{\alpha N} & {\left[\frac{N_{1}}{2} d\left(h_{\mu}^{\mu}-h_{a}^{a}+h_{i}^{i}\right) \wedge \operatorname{vol}_{A d S_{4}} \wedge \operatorname{vol}_{B_{3}}\right.} \\
& \left.-\frac{N_{2}}{2} d\left(h_{\mu}^{\mu}+h_{a}^{a}-h_{i}^{i}\right) \wedge \operatorname{vol}_{A d S_{4}} \wedge \operatorname{vol}_{A_{3}}\right] .
\end{aligned}
$$

Then we can again write $h_{3}=d b_{2}$, and expand the fluctuation fields in terms of spherical harmonics on $A_{3}$ and $B_{3}$. This gives rise to the following set of equations:

$$
\begin{aligned}
& d\left[\left\{\frac{N_{1}}{2}\left(H^{I J}-3 \pi_{(1)}^{I J}-\pi_{(2)}^{I J}\right)-N_{1} \delta \phi^{I J}-\frac{\alpha^{2} N^{2}}{Q^{2}} Q_{1} \delta C_{0}^{I J}\right.\right. \\
& \left.\left.\quad+\left(\square_{x}+\square_{y}+\square_{z}\right) b_{(1)}^{I J}\right\} Y^{I} Z^{J}\right] \wedge \operatorname{vol}_{A d S_{4}} \wedge \operatorname{vol}_{B_{3}}=0,
\end{aligned}
$$

\footnotetext{
${ }^{8}$ We use the following notation: $\square_{x} \equiv g^{\mu \nu} \nabla_{\mu} \nabla_{\nu}, \square_{y} \equiv g^{a b} \nabla_{a} \nabla_{b}$, and $\square_{z} \equiv g^{i j} \nabla_{i} \nabla_{j}$.
} 


$$
\begin{aligned}
d[ & \left\{\frac{N_{2}}{2}\left(H^{I J}-\pi_{(1)}^{I J}-3 \pi_{(2)}^{I J}\right)-N_{2} \delta \phi^{I J}-\frac{\alpha^{2} N^{2}}{Q^{2}} Q_{2} \delta C_{0}^{I J}\right. \\
& \left.\left.+\left(\square_{x}+\square_{y}+\square_{z}\right) b_{(2)}^{I J}\right\} Y^{I} Z^{J}\right] \wedge \operatorname{vol}_{A d S_{4}} \wedge \operatorname{vol}_{A_{3}}=0 .
\end{aligned}
$$

Similarly one can derive the fluctuation equations for $F_{3}$ :

$$
\begin{aligned}
& d\left[\left\{\frac{Q_{1}}{2}\left(H^{I J}-3 \pi_{(1)}^{I J}-\pi_{(2)}^{I J}\right)+Q_{1} \delta \phi^{I J}-N_{1} \delta C_{0}^{I J}\right.\right. \\
& \left.\left.\quad+\left(\square_{x}+\square_{y}+\square_{z}\right) c_{(1)}^{I J}\right\} Y^{I} Z^{J}\right] \wedge \operatorname{vol}_{A d S_{4}} \wedge \operatorname{vol}_{B_{3}}=0 \\
& d\left[\left\{\frac{Q_{2}}{2}\left(H^{I J}-\pi_{(1)}^{I J}-3 \pi_{(2)}^{I J}\right)+Q_{2} \delta \phi^{I J}-N_{2} \delta C_{0}^{I J}\right.\right. \\
& \left.\left.\quad+\left(\square_{x}+\square_{y}+\square_{z}\right) c_{(2)}^{I J}\right\} Y^{I} Z^{J}\right] \wedge \operatorname{vol}_{A d S_{4}} \wedge \operatorname{vol}_{A_{3}}=0
\end{aligned}
$$

Let us now consider Einstein's equations to linear order in fluctuations. We shall need to expand the Ricci tensor to linear order; in our conventions, this is:

$$
\begin{aligned}
\delta R_{M N}=- & \frac{1}{2}\left[\left(\square_{x}+\square_{y}+\square_{z}\right) h_{M N}+\nabla_{M} \nabla_{N} h_{P}^{P}-\nabla_{M} \nabla^{P} h_{P N}-\nabla_{N} \nabla^{P} h_{P M}\right. \\
& \left.-2 R_{M P Q N} h^{P Q}-R_{M}^{P} h_{N P}-R_{N}^{P} h_{M P}\right] .
\end{aligned}
$$

In terms of the graviton modes defined in Eqs. (28)-(30), the components of $\delta R_{M N}$ with both indices in the internal manifold are written as

$$
\begin{aligned}
\delta R_{a b}=-\frac{1}{2}[ & \left(\square_{x}+\square_{y}+\square_{z}\right) h_{(a b)}-2 R_{a c d b} h^{(c d)}-R_{a}{ }^{c} h_{(b c)}-R_{b}{ }^{c} h_{(a c)} \\
& +\frac{1}{3} g_{a b}\left(\square_{x}+\square_{y}+\square_{z}\right) h_{c}^{c}+\nabla_{a} \nabla_{b}\left(H_{\mu}^{\mu}-\frac{5}{3} h_{c}^{c}-h_{i}^{i}\right) \\
& \left.-\nabla_{a} \nabla^{\mu} h_{\mu b}-\nabla_{b} \nabla^{\mu} h_{\mu a}\right], \\
\delta R_{i j}=-\frac{1}{2}\left[\left(\square_{x}+\square_{y}+\square_{z}\right) h_{(i j)}-2 R_{i k l j} h^{(k l)}-R_{i}{ }^{k} h_{(j k)}-R_{j}{ }^{k} h_{(i k)}\right. & \\
& +\frac{1}{3} g_{i j}\left(\square_{x}+\square_{y}+\square_{z}\right) h_{k}^{k}+\nabla_{i} \nabla_{j}\left(H_{\mu}^{\mu}-h_{c}^{c}-\frac{5}{3} h_{k}^{k}\right) \\
& \left.-\nabla_{i} \nabla^{\mu} h_{\mu j}-\nabla_{j} \nabla^{\mu} h_{\mu i}\right] .
\end{aligned}
$$

We have used the gauge conditions (32) to simplify some of the terms in the above expressions. 
On the other hand, the variation of the stress-energy tensor $\bar{T}_{M N}=T_{M N}-\frac{1}{8} g_{M N} T_{P}^{P}$ is given as follows:

$$
\begin{aligned}
& \delta \bar{T}_{a b}=e^{-\phi}\left[-\left(\frac{1}{2 \cdot 2 !} H_{a c d} H_{b}{ }^{c d}-\frac{2}{2 \cdot 8} g_{a b}\left|H_{3}\right|^{2}\right) \delta \phi\right. \\
& +\frac{1}{2 \cdot 2 !}\left(h_{a c d} H_{b}{ }^{c d}+H_{a c d} h_{b}{ }^{c d}-2 h^{c d} H_{a c e} H_{b d}{ }^{e}\right)-\frac{2}{2 \cdot 8} h_{a b}\left|H_{3}\right|^{2} \\
& \left.-\frac{2}{2 \cdot 8} g_{a b}\left(\frac{2}{3 !} H^{c d e} h_{c d e}+\frac{2}{3 !} H^{i j k} h_{i j k}-\left(N_{1}^{2} h_{c}^{c}+N_{2}^{2} h_{i}^{i}\right)\right)\right] \\
& +e^{\phi}\left[\left(\frac{1}{2 \cdot 2 !} F_{a c d} F_{b}{ }^{c d}-\frac{2}{2 \cdot 8} g_{a b}\left|F_{3}\right|^{2}\right) \delta \phi\right. \\
& +\frac{1}{2 \cdot 2 !}\left(f_{a c d} F_{b}{ }^{c d}+F_{a c d} f_{b}{ }^{c d}-2 h^{c d} F_{a c e} F_{b d}{ }^{e}\right)-\frac{2}{2 \cdot 8} h_{a b}\left|F_{3}\right|^{2} \\
& \left.-\frac{2}{2 \cdot 8} g_{a b}\left(\frac{2}{3 !} F^{c d e} f_{c d e}+\frac{2}{3 !} F^{i j k} f_{i j k}-\left(Q_{1}^{2} h_{c}^{c}+Q_{2}^{2} h_{i}^{i}\right)\right)\right] \\
& -e^{\phi}\left[\frac{1}{2 \cdot 2 !} H_{a c d} F_{b}{ }^{c d}+\frac{1}{2 \cdot 2 !} F_{a c d} H_{b}{ }^{c d}\right] \delta C_{0}+\kappa_{10}^{2} \delta \bar{T}_{a b}^{\mathrm{loc}}, \\
& \delta \bar{T}_{i j}=e^{-\phi}\left[-\left(\frac{1}{2 \cdot 2 !} H_{i k l} H_{j}{ }^{k l}-\frac{2}{2 \cdot 8} g_{i j}\left|H_{3}\right|^{2}\right) \delta \phi\right. \\
& +\frac{1}{2 \cdot 2 !}\left(h_{i k l} H_{j}{ }^{k l}+H_{i k l} h_{j}{ }^{k l}-2 h^{k l} H_{i k m} H_{j l}{ }^{m}\right)-\frac{2}{2 \cdot 8} h_{i j}\left|H_{3}\right|^{2} \\
& \left.-\frac{2}{2 \cdot 8} g_{i j}\left(\frac{2}{3 !} H^{a b c} h_{a b c}+\frac{2}{3 !} H^{k l m} h_{k l m}-\left(N_{1}^{2} h_{a}^{a}+N_{2}^{2} h_{k}^{k}\right)\right)\right] \\
& +e^{\phi}\left[\left(\frac{1}{2 \cdot 2 !} F_{i k l} F_{j}^{k l}-\frac{2}{2 \cdot 8} g_{i j}\left|F_{3}\right|^{2}\right) \delta \phi\right. \\
& +\frac{1}{2 \cdot 2 !}\left(f_{i k l} F_{j}{ }^{k l}+F_{i k l} f_{j}{ }^{k l}-2 h^{k l} F_{i k m} F_{j l}{ }^{m}\right)-\frac{2}{2 \cdot 8} h_{i j}\left|F_{3}\right|^{2} \\
& \left.-\frac{2}{2 \cdot 8} g_{i j}\left(\frac{2}{3 !} F^{a b c} f_{a b c}+\frac{2}{3 !} F^{k l m} f_{k l m}-\left(Q_{1}^{2} h_{a}^{a}+Q_{2}^{2} h_{k}^{k}\right)\right)\right] \\
& -e^{\phi}\left[\frac{1}{2 \cdot 2 !} H_{i k l} F_{j}{ }^{k l}+\frac{1}{2 \cdot 2 !} F_{i k l} H_{j}{ }^{k l}\right] \delta C_{0}+\kappa_{10}^{2} \delta \bar{T}_{i j}^{\text {loc }} .
\end{aligned}
$$

The contributions $\delta \bar{T}_{a b}^{\text {loc }}$ and $\delta \bar{T}_{i j}^{\text {loc }}$ from O3-planes are calculated in the following way. Recall from Eq. (15) that

$$
\kappa_{10}^{2} \bar{T}_{a b}^{\mathrm{loc}}=-\frac{\kappa_{10}^{2} T_{3} \rho}{8} g_{a b},
$$

where $-T_{3} / 4$ is the tension of an O3-plane, and $\rho$ is their density. By considering the metric fluctuation in the internal space and the resulting fluctuation of $\rho$, we obtain

$$
\kappa_{10}^{2} g^{i j} \delta \bar{T}_{i j}^{\mathrm{loc}}=\frac{N Q}{16}\left(\alpha+\frac{1}{\alpha}\right)\left(\pi_{1}+3 \pi_{2}\right), \quad \kappa_{10}^{2} g^{a b} \delta \bar{T}_{a b}^{\mathrm{loc}}=\frac{N Q}{16}\left(\alpha+\frac{1}{\alpha}\right)\left(3 \pi_{1}+\pi_{2}\right)
$$


where we have used $2 \kappa_{10}^{2} T_{3}=(2 \pi)^{4} \alpha^{\prime 2}$.

Now we equate $\delta R_{M N}$ with $\delta \bar{T}_{M N}$ and expand each fluctuation mode in terms of the spherical harmonics. The trace part of (50) and (52) then reads

$$
\begin{aligned}
& {\left[\left\{\square_{x}-\frac{2}{3} \square_{y}+\square_{z}-\frac{13}{8}\left(\alpha+\frac{1}{\alpha}\right) N Q\right\} \pi_{(1)}^{I J}+\left\{-\square_{y}+\frac{9}{8}\left(\alpha+\frac{1}{\alpha}\right) N Q\right\} \pi_{(2)}^{I J}\right.} \\
& +\square_{y} H^{I J}-3 N Q\left(\alpha-\frac{1}{\alpha}\right) \delta \phi^{I J}+6 \alpha N^{2} \delta C_{0}^{I J}+\frac{Q}{\alpha N}\left(\frac{9}{2} N_{1} \square_{y} b_{(1)}^{I J}-\frac{3}{2} N_{2} \square_{z} b_{(2)}^{I J}\right) \\
& \left.+\frac{\alpha N}{Q}\left(\frac{9}{2} Q_{1} \square_{y} c_{(1)}^{I J}-\frac{3}{2} Q_{2} \square_{z} c_{(2)}^{I J}\right)\right] Y^{I} Z^{J}=0,
\end{aligned}
$$

while the traceless part $\delta R_{(a b)}=\delta \bar{T}_{(a b)}$ gives the following equation:

$$
\left(H^{I J}-\frac{5}{3} \pi_{(1)}^{I J}-\pi_{(2)}^{I J}\right) \nabla_{(a} \nabla_{b)} Y^{I} Z^{J}=0
$$

We get similar set of equations from (51) and (53):

$$
\begin{aligned}
& {\left[\left\{\square_{x}+\square_{y}-\frac{2}{3} \square_{z}-\frac{13}{8}\left(\alpha+\frac{1}{\alpha}\right) N Q\right\} \pi_{(2)}^{I J}+\left\{-\square_{z}+\frac{9}{8}\left(\alpha+\frac{1}{\alpha}\right) N Q\right\} \pi_{(1)}^{I J}\right.} \\
& +\square_{z} H^{I J}+3 N Q\left(\alpha-\frac{1}{\alpha}\right) \delta \phi^{I J}-6 \alpha N^{2} \delta C_{0}^{I J}-\frac{Q}{\alpha N}\left(\frac{3}{2} N_{1} \square_{y} b_{(1)}^{I J}-\frac{9}{2} N_{2} \square_{z} b_{(2)}^{I J}\right) \\
& \left.\quad-\frac{\alpha N}{Q}\left(\frac{3}{2} Q_{1} \square_{y} c_{(1)}^{I J}-\frac{9}{2} Q_{2} \square_{z} c_{(2)}^{I J}\right)\right] Y^{I} Z^{J}=0,
\end{aligned}
$$

and

$$
\left(H^{I J}-\pi_{(1)}^{I J}-\frac{5}{3} \pi_{(2)}^{I J}\right) Y^{I} \nabla_{(i} \nabla_{j)} Z^{J}=0
$$

\subsection{Mass Spectrum}

So far in this section, we derived the fluctuation equations of motion assuming that the internal manifold is $A_{3} \times B_{3}$ without the orientifold action. But as we saw in Section 2. the tadpole constraint (11) requires the presence of O3-planes in the solution. We now discuss how this will affect the mass spectrum.

The orientifold action can be written as $\Omega(-1)^{F_{L}} R$, where $R$ is a $\mathbf{Z}_{2}$ action on the internal manifold and $\Omega(-1)^{F_{L}}$ takes the value +1 on the metric $g_{M N}, \mathrm{R}-\mathrm{R}$ 4-form $C_{4}$, axion $C_{0}$ and dilaton $\phi$, and -1 on the NS-NS and R-R 2-form potentials $B_{2}$ and $C_{2}$ [32, 33]. This implies that we want our metric, axion, and dilaton fluctuations to transform by +1 under the $\mathbf{Z}_{2}$ action $R$, and NS-NS and R-R 2-form fluctuations to transform by -1 . We shall consider a specific type of $\mathbf{Z}_{2}$ action $R=R_{1} \times R_{2}:(y, z) \mapsto$ 
$\left(y^{\prime}, z^{\prime}\right)$ on $A_{3} \times B_{3}$, where $R_{1}: y \mapsto y^{\prime}$ and $R_{2}: z \mapsto z^{\prime}$ are $\mathbf{Z}_{2}$ symmetries of $A_{3}$ and and $B_{3}$, respectively. Then our background metric (44) and dilaton expectation value (8) are obviously consistent with the orientifold action.

To see the consistency of our background $H_{3}$ and $F_{3}$ choices, we need to consider how they transform under the $\mathbf{Z}_{2}$ action $R$. Let us first consider $H_{a b c}(y)=N_{1} \epsilon_{a b c}(y)$. The transformation property of $B_{a b}$ under $R$ can be written as

$$
B_{a b}\left(y^{\prime}\right)=-\frac{\partial y^{c}}{\partial y^{\prime a}} \frac{\partial y^{d}}{\partial y^{\prime b}} B_{c d}(y) \text {. }
$$

This implies

$$
\begin{aligned}
H_{a b c}\left(y^{\prime}\right) & =\partial_{a} B_{b c}\left(y^{\prime}\right)+\text { cyclic permutations in } a, b, c \\
& =\frac{\partial}{\partial y^{\prime a}}\left[-\frac{\partial y^{d}}{\partial y^{\prime b}} \frac{\partial y^{e}}{\partial y^{\prime c}} B_{d e}(y)\right]+\text { permutations } \\
& =-\frac{\partial y^{d}}{\partial y^{\prime b}} \frac{\partial y^{e}}{\partial y^{\prime} c} \frac{\partial y^{f}}{\partial y^{\prime a}} \frac{\partial B_{d e}(y)}{\partial y^{f}}+\text { permutations } \\
& =-\frac{\partial y^{f}}{\partial y^{\prime a}} \frac{\partial y^{d}}{\partial y^{\prime b}} \frac{\partial y^{e}}{\partial y^{\prime c}} H_{f d e}(y)=-\left( \pm N_{1} \epsilon_{a b c}\left(y^{\prime}\right)\right),
\end{aligned}
$$

where the sign in the last line depends on whether $R_{1}$ is orientation-preserving or not. Therefore, we see that the background $H_{a b c}(y)=N_{1} \epsilon_{a b c}(y)$ is consistent with the orientifold action only if $R_{1}$ is an orientation-reversing map on $A_{3}$. Similarly, $H_{i j k}=N_{2} \epsilon_{i j k}(z)$ is a consistent background only if $R_{2}$ is orientation-reversing on $B_{3}$. The same holds true for $F_{3}$ background values.

Having shown that both $R_{1}$ and $R_{2}$ must be orientation-reversing maps on respective manifolds, we determine which fluctuation modes will survive. We can choose our spherical harmonics $Y^{I}(y)$ and $Z^{J}(z)$ to be the parity eigenmodes under $R_{1}$ and $R_{2}$ :

$$
Y^{I}\left(y^{\prime}\right)= \pm Y^{I}(y), \quad Z^{J}\left(z^{\prime}\right)= \pm Z^{J}(z) \text {. }
$$

We shall denote the parity eigenvalues by $(-1)^{I}$ and $(-1)^{J}$, respectively, and call the indices $I$ and $J$ even or odd depending on the parity of $Y^{I}(y)$ and $Z^{J}(z)$. From the mode expansion given in Eqs. (34)-(38), it is then clear that for the fluctuations $H^{I J}$, $\pi_{(1),(2)}^{I J}, \delta \phi^{I J}$, and $\delta C_{0}^{I J}$, the modes that survive the orientifold projection are those with $I$ and $J$ both even or both odd. Things are slightly trickier for the 2-form potential fluctuations $b_{2}$ and $c_{2}$. For example, let's consider the fluctuation $b_{a b}$, whose mode expansion is given in Eq. (35). Under the action of $R$, it transforms as

$$
\begin{aligned}
b_{(1)}^{I J}(x) \epsilon_{a b}{ }^{c}(y) & \nabla_{c} Y^{I}(y) Z^{J}(z) \rightarrow \frac{\partial y^{\prime} c}{\partial y^{a}} \frac{\partial y^{\prime} d}{\partial y^{b}} b_{(1)}^{I J}(x) \epsilon_{c d}{ }^{e}\left(y^{\prime}\right) \nabla_{e} Y^{I}\left(y^{\prime}\right) Z^{J}\left(z^{\prime}\right) \\
& =b_{(1)}^{I J}(x) \frac{\partial y^{\prime} c}{\partial y^{a}} \frac{\partial y^{\prime} d}{\partial y^{b}} \epsilon_{c d}{ }^{e}\left(y^{\prime}\right) \frac{\partial y^{f}}{\partial y^{\prime} e} \frac{\partial}{\partial y^{f}}(-1)^{I} Y(y)(-1)^{J} Z^{J}(z) \\
& =-b_{(1)}^{I J}(x) \epsilon_{a b}{ }^{f}(y)(-1)^{I} \nabla_{f} Y(y)(-1)^{J} Z^{J}(z),
\end{aligned}
$$


where the minus sign in the third line comes from the fact that $R_{1}$ is orientationreversing. Since the R-R 2-form should transform by -1 under $R$, the modes $b_{(1)}^{I J}$ that survive the projection are those with $I$ and $J$ both even or both odd. The cases for $b_{i j}, c_{a b}$, and $c_{i j}$ are all exactly the same. In conclusion, for all the fluctuation modes given in Eqs. (34)-(38), those which survive the orientifold projection are precisely those with $I$ and $J$ both even or both odd.

Now we compute the spectrum of the fluctuation modes that survive the orientifold projection and determine the stability of our background. Let us first introduce some notations. We denote the eigenvalues of the Laplacians on $A_{3}$ and $B_{3}$ as follows:

$$
\square_{y} Y^{I}=-\frac{\lambda^{I}}{R^{2}} Y^{I}, \quad \square_{z} Z^{J}=-\frac{\mu^{J}}{R^{2}} Z^{J}
$$

For the lowest harmonics on the respective manifolds, that is, $Y^{I}(y)=$ constant and $Z^{J}(z)=$ constant, the corresponding eigenvalues are obviously $\lambda^{I}=\mu^{J}=0$. For non-constant $Y^{I}$ and $Z^{J}$, they have a lower bound $\lambda^{I}, \mu^{J} \geq 3$, which holds for any 3-dimensional compact Einstein manifold of positive curvature [46]. We also find it convenient to re-scale the 2 -form potential fluctuations as follows:

$$
\begin{array}{rlrl}
b_{(1)}^{I J}=\frac{1}{\alpha} N_{1} L^{2} B_{(1)}^{I J}, & b_{(2)} & =\alpha N_{2} L^{2} B_{(2)}^{I J}, \\
c_{(1)}^{I J}=\alpha Q_{1} L^{2} C_{(1)}^{I J}, & c_{(2)}^{I J}=\frac{1}{\alpha} Q_{2} L^{2} C_{(2)}^{I J} .
\end{array}
$$

We start with the lowest harmonics on both manifolds. From Eqs. (35) and (36), we see that there are no form field fluctuations at this level, as they involve derivatives on $Y^{I}$ and $Z^{J}$. But all the other fluctuations survive orientifold projection because $Y^{I}$ and $Z^{J}$, both being constant, are obviously even under the $\mathbf{Z}_{2}$ action. Using the fact that $\square_{y} Y^{I}=0$ and $\square_{z} Z^{J}=0$, the contents of dilaton equation (41), axion equation (42), and metric equations (56) and (58) can be summarized as follows:

$$
L^{2} \square_{x}\left(\begin{array}{c}
\delta \phi \\
\delta C_{0} \\
\pi_{(1)} \\
\pi_{(2)}
\end{array}\right)=\left(\begin{array}{cccc}
24 & 0 & \frac{12\left(\alpha^{2}-1\right)}{\alpha^{2}+1} & -\frac{12\left(\alpha^{2}-1\right)}{\alpha^{2}+1} \\
0 & 24 & -\frac{24 \alpha}{\left(\alpha^{2}+1\right) g_{s}} & \frac{24 \alpha}{\left(\alpha^{2}+1\right) g_{s}} \\
\frac{72\left(\alpha^{2}-1\right)}{\alpha^{2}+1} & -\frac{144 \alpha g_{s}}{\alpha^{2}+1} & 39 & -27 \\
-\frac{72\left(\alpha^{2}-1\right)}{\alpha^{2}+1} & \frac{144 \alpha g_{s}}{\alpha^{2}+1} & -27 & 39
\end{array}\right)\left(\begin{array}{c}
\delta \phi \\
\delta C_{0} \\
\pi_{(1)} \\
\pi_{(2)}
\end{array}\right) .
$$

Here we have used the relations (81) and (19) for our background. All the other fluctuation equations of motion are trivially satisfied because $\nabla_{a} Y^{I}=\nabla_{i} Z^{J}=0$. The eigenvalues of the mass matrix are

$$
m^{2} L^{2}=12,24,3(15 \pm \sqrt{241}) .
$$

Even though one of them has negative mass-squared, they are all above the BF bound (27), and the background is stable against this fluctuation mode. 
Next, we consider the modes where one of the harmonics $Y^{I}$ and $Z^{J}$ is constant but the other is a higher harmonic. For definiteness, let us assume that $\nabla_{a} Y^{I}=0$ but $\nabla_{i} Z^{J} \neq 0$. Then the fluctuation $b_{(1)}^{I J}$ and $c_{(1)}^{I J}$ are still absent, but we now have $b_{(2)}^{I J}$ and $c_{(2)}^{I J}$ for even $J$ (because $I$ is even). All the other fluctuations are also present for even $J$. Since $\nabla_{(i} \nabla_{j)} Z^{J} \neq 0$ for nonconstant $Z^{J}$ on a generic ${ }^{9} 3$-manifold $B_{3}$, Eq. (59) gives an algebraic constraint

$$
H^{I J}-\pi_{(1)}^{I J}-\frac{5}{3} \pi_{(2)}^{I J}=0,
$$

which we can use to eliminate $H^{I J}$ in Eq. (58) in favor of $\pi_{(1)}$ and $\pi_{(2)}$. Using $\square_{y} Y^{I}=0$ and $\square_{z} Z^{J}=-\left(\mu^{J} / R^{2}\right) Z^{J}$, the fluctuation equations (41), (42), (46), (48), (56), and (58) result in the following mass matrix:

$$
L^{2} \square_{x}\left(\begin{array}{c}
\delta \phi \\
\delta C_{0} \\
B_{(2)} \\
C_{(2)} \\
\pi_{(1)} \\
\pi_{(2)}
\end{array}\right)=M^{2}\left(\begin{array}{c}
\delta \phi \\
\delta C_{0} \\
B_{(2)} \\
C_{(2)} \\
\pi_{(1)} \\
\pi_{(2)}
\end{array}\right)
$$

where

$$
M^{2}=\left(\begin{array}{cccccc}
\frac{3}{2} \mu^{J}+24 & 0 & \frac{36 \alpha \mu^{J}}{\alpha^{2}+1} & -\frac{36 \alpha \mu^{J}}{\alpha^{2} J^{1}} & \frac{12\left(\alpha^{2}-1\right)}{\alpha^{2}+1} & -\frac{12\left(\alpha^{2}-1\right)}{\alpha^{2}+1} \\
0 & \frac{3}{2} \mu^{J}+24 & \frac{36 \alpha^{2} \mu^{J}}{\left(\alpha^{2}+1\right) g_{s}} & \frac{36 \sigma^{J}}{\left(\alpha^{2}+1\right) g_{s}} & -\frac{24 \alpha}{\left(\alpha^{2}+1\right) g_{s}} & \frac{24 \alpha}{\left(\alpha^{2}+1\right) g_{s}} \\
\frac{1}{\alpha} & g_{s} & \frac{3}{2} \mu^{J} & 0 & 0 & \frac{2}{3 \alpha} \\
-\alpha & g_{s} & 0 & \frac{3}{2} \mu^{J} & 0 & \frac{2 \alpha}{3} \\
\frac{72\left(\alpha^{2}-1\right)}{\alpha^{2}+1} & -\frac{144 \alpha g_{s}}{\alpha^{2}+1} & -\frac{54 \alpha \mu^{J}}{\alpha^{2}+1} & -\frac{54 \alpha \mu^{J}}{\alpha^{2}+1} & \frac{3}{2} \mu^{J}+39 & -27 \\
-\frac{72\left(\alpha^{2}-1\right)}{\alpha^{2}+1} & \frac{144 g_{s}}{\alpha^{2}+1} & \frac{162 \alpha \mu^{J}}{\alpha^{2}+1} & \frac{162 \mu^{J}}{\alpha^{2}+1} & -27 & \frac{3}{2} \mu^{J}+39
\end{array}\right) .
$$

The expression for the eigenvalues of (69) in terms of $\mu^{J}$ is quite complicated, but it is independent of $\alpha$ and $g_{s}$. One also finds that the smallest eigenvalue lies below the BF bound if $3 \leq \mu^{J} \lesssim 22.1$. That is, the background is unstable if $B_{3}$ has a non-constant even scalar harmonic with $\mu^{J} \lesssim 22.1$. Exchanging the roles of $A_{3}$ and $B_{3}$ yields exactly same result: the background is unstable if $A_{3}$ has a non-constant scalar harmonic with $\lambda^{I} \lesssim 22.1$

Assuming that non-zero eigenvalues of the Laplacian on $A_{3}$ and $B_{3}$ are all larger than $\sim 22.1$, we continue to check the modes with both $Y^{I}$ and $Z^{J}$ non-constant. For

\footnotetext{
${ }^{9}$ This does not hold when $B_{3}$ is maximally symmetric, i.e. a 3 -sphere. In this case, the eigenvalues of the Laplacian are given by $\mu^{J}=k(k+2)$, where $k=0,1,2, \ldots$, and $\nabla_{(i} \nabla_{j)} Z^{J}=0$ not only for $k=0$ mode but also for $k=1$ modes. Therefore, the algebraic constraint in (67) holds only for $k \geq 2$ modes.
} 
$I$ and $J$ both even or both odd, we obtain another algebraic constraint from Eq. (59) which is analogous to Eq. (67). Together, these two constraints imply

$$
\pi_{(1)}^{I J}=\pi_{(2)}^{I J} \equiv \pi^{I J} \quad \text { and } \quad H^{I J}=\frac{8}{3} \pi^{I J},
$$

which allows us to eliminate $H^{I J}$ and $\pi_{(1),(2)}^{I J}$ in favor of $\pi^{I J}$. The mass matrix we get from the fluctuation equations is then

$$
L^{2} \square_{x}\left(\begin{array}{c}
\delta \phi \\
\delta C_{0} \\
B_{(1)} \\
B_{(2)} \\
C_{(1)} \\
C_{(2)} \\
\pi
\end{array}\right)=M^{2}\left(\begin{array}{c}
\delta \phi \\
\delta C_{0} \\
B_{(1)} \\
B_{(2)} \\
C_{(1)} \\
C_{(2)} \\
\pi
\end{array}\right),
$$

where $M^{2}$ is now

$$
\left(\begin{array}{ccccccc}
\frac{3\left(\lambda^{I}+\mu^{J}\right)+48}{2} & 0 & \frac{36 \alpha \lambda^{I}}{\alpha^{2}+1} & \frac{36 \alpha \mu^{J}}{\alpha^{2}+1} & -\frac{36 \alpha \lambda^{I}}{\alpha^{2}+1} & -\frac{36 \alpha \mu^{J}}{\alpha^{2}+1} & 0 \\
0 & \frac{3\left(\lambda^{I}+\mu^{J}\right)+48}{2} & -\frac{36 \lambda^{I}}{\left(\alpha^{2}+1\right) g_{s}} & \frac{36 \alpha^{2} \mu^{J}}{\left(\alpha^{2}+1\right) g_{s}} & -\frac{36 \alpha^{2} \lambda^{I}}{\left(\alpha^{2}+1\right) g_{s}} & \frac{36 \mu^{J}}{\left(\alpha^{2}+1\right) g_{s}} & 0 \\
\alpha & -g_{s} & \frac{3\left(\lambda^{I}+\mu^{J}\right)}{2} & 0 & 0 & 0 & \frac{2 \alpha}{3} \\
\frac{1}{\alpha} & g_{s} & 0 & \frac{3\left(\lambda^{I}+\mu^{J}\right)}{2} & 0 & 0 & \frac{2}{3 \alpha} \\
-\frac{1}{\alpha} & -g_{s} & 0 & 0 & \frac{3\left(\lambda^{I}+\mu^{J}\right)}{2} & 0 & \frac{2}{3 \alpha} \\
-\alpha & g_{s} & 0 & 0 & 0 & \frac{3\left(\lambda^{I}+\mu^{J}\right)}{2} & \frac{2 \alpha}{3} \\
0 & 0 & \frac{54 \alpha \lambda^{I}}{\alpha^{2}+1} & \frac{54 \alpha \mu^{J}}{\alpha^{2}+1} & \frac{54 \alpha \lambda^{I}}{\alpha^{2}+1} & \frac{54 \alpha \mu^{J}}{\alpha^{2}+1} & \frac{3\left(\lambda^{I}+\mu^{J}\right)+24}{2}
\end{array}\right) .
$$

Again, the eigenvalues are quite complicated functions of $\lambda^{I}$ and $\mu^{J}$, but numerical experimentation shows that they are all above the BF bound for $\lambda^{I}, \mu^{J} \gtrsim 22.1$.

From these observations, we conclude that the criterion for the stability of these Freund-Rubin compactifications is that there be no even eigenfunction of the scalar Laplacian with eigenvalue between 3 and 22.1.

We end this section with the simplest example of this kind where we take both $A_{3}$ and $B_{3}$ to be 3 -spheres. For each 3 -sphere, we take the coordinate system $(\psi, \theta, \phi)$ defined by

$$
\begin{array}{ll}
x=R \cos \psi, & y=R \sin \psi \cos \theta, \\
z=R \sin \psi \sin \theta \cos \phi, & w=R \sin \psi \sin \theta \sin \phi,
\end{array}
$$

in terms of the 3 -sphere $x^{2}+y^{2}+z^{2}+w^{2}=R^{2}$ embedded in $\mathbb{R}^{4}$. The orientationreversing $\mathbf{Z}_{2}$ action on $S^{3}$ is defined as

$$
\psi \rightarrow \pi-\psi, \quad \theta \rightarrow \pi-\theta, \quad \phi \rightarrow 2 \pi-\phi .
$$


It has two fixed points on each 3-sphere, so the number of O3-planes on $S^{3} \times S^{3}$ is four. The eigenvalues of the Laplacian on $S^{3}$ are given by $\mu^{J}=k(k+2)$ where $k=0,1,2, \ldots$, and we obtain the mass matrix Eq. (68) when $k=0$ on the first 3 -sphere and $k \geq 2$ on the second. In particular, for $k=2$ and $k=3$ on the second 3 -sphere, the corresponding eigenvalues are $\mu^{J}=8$ and 15 . Since both $k=2$ and $k=3$ have modes which are even under (72), this background is unstable against small fluctuations.

\section{Acknowledgements}

We would like to thank M. Douglas, O. Ganor, P. Horava, S. Kachru, I. Klebanov, L. McAllister, J. McGreevy, W. Taylor and especially O. deWolfe and E. Silverstein for helpful discussions. We would also like to thank T. Shiromizu for a helpful correspondence. Y. P. H would like to thank the organizers and participants of TASI 2005 for hospitality and helpful discussions. I. M. was supported in part by the Director, Office of Science, Office of High Energy and Nuclear Physics, of the U.S. Department of Energy under Contract DE-AC02-05CH11231, in part by the National Science Foundation under grant PHY-00-98840, and by the Berkeley Center for Theoretical Physics. I. M. would also like to thank the Aspen Center for Physics for hospitality during the final stages of this work.

\section{References}

[1] S. Kachru, R. Kallosh, A. Linde, and S. P. Trivedi, "De Sitter vacua in string theory," Phys. Rev. D68 (2003) 046005, hep-th/0301240.

[2] O. DeWolfe, A. Giryavets, S. Kachru, and W. Taylor, "Type IIA moduli stabilization," JHEP 07 (2005) 066, hep-th/0505160.

[3] O. DeWolfe, A. Giryavets, S. Kachru, and W. Taylor, "Enumerating flux vacua with enhanced symmetries," JHEP 02 (2005) 037, hep-th/0411061.

[4] O. DeWolfe, "Enhanced symmetries in multiparameter flux vacua," hep-th/0506245.

[5] F. Denef, M. R. Douglas, B. Florea, A. Grassi, and S. Kachru, "Fixing all moduli in a simple F-theory compactification," hep-th/0503124.

[6] J. P. Conlon, F. Quevedo, and K. Suruliz, "Large-volume flux compactifications: Moduli spectrum and D3/D7 soft supersymmetry breaking," hep-th/0505076. 
[7] V. Balasubramanian, P. Berglund, J. P. Conlon, and F. Quevedo, "Systematics of moduli stabilisation in Calabi-Yau flux compactifications," JHEP 03 (2005) 007, hep-th/0502058.

[8] B. S. Acharya, "A moduli fixing mechanism in M theory," hep-th/0212294.

[9] G. Curio, A. Krause, and D. Lust, "Moduli stabilization in the heterotic / IIB discretuum," hep-th/0502168.

[10] K. Becker, M. Becker, K. Dasgupta, and P. S. Green, "Compactifications of heterotic theory on non-Kaehler complex manifolds. I," JHEP 04 (2003) 007, hep-th/0301161.

[11] K. Becker, M. Becker, P. S. Green, K. Dasgupta, and E. Sharpe, "Compactifications of heterotic strings on non-Kaehler complex manifolds. II," Nucl. Phys. B678 (2004) 19-100, hep-th/0310058.

[12] R. Brustein and S. P. de Alwis, "Moduli potentials in string compactifications with fluxes: Mapping the discretuum," Phys. Rev. D69 (2004) 126006, hep-th/0402088.

[13] J. Shelton, W. Taylor, and B. Wecht, "Nongeometric Flux Compactifications," hep-th/0508133.

[14] I. Antoniadis and T. Maillard, "Moduli stabilization from magnetic fluxes in type I string theory," Nucl. Phys. B716 (2005) 3-32, hep-th/0412008.

[15] I. Antoniadis, A. Kumar, and T. Maillard, "Moduli stabilization with open and closed string fluxes," hep-th/0505260

[16] G. Curio and A. Krause, "G-fluxes and non-perturbative stabilisation of heterotic M- theory," Nucl. Phys. B643 (2002) 131-156, hep-th/0108220.

[17] R. Bousso and J. Polchinski, "Quantization of four-form fluxes and dynamical neutralization of the cosmological constant," JHEP 06 (2000) 006, hep-th/0004134.

[18] M. R. Douglas, "The statistics of string / M theory vacua," JHEP 05 (2003) 046, hep-th/0303194.

[19] S. Ashok and M. R. Douglas, "Counting flux vacua," JHEP 01 (2004) 060, hep-th/0307049.

[20] F. Denef and M. R. Douglas, "Distributions of flux vacua," JHEP 05 (2004) 072, hep-th/0404116. 
[21] L. Susskind, "The anthropic landscape of string theory," hep-th/0302219.

[22] M. R. Douglas, "Statistical analysis of the supersymmetry breaking scale," hep-th/0405279.

[23] R. Bousso, O. DeWolfe, and R. C. Myers, "Unbounded entropy in spacetimes with positive cosmological constant," Found. Phys. 33 (2003) 297-321, hep-th/0205080.

[24] C. Krishnan, S. Paban, and M. Zanic, "Evolution of gravitationally unstable de Sitter compactifications," JHEP 05 (2005) 045, hep-th/0503025.

[25] P. G. O. Freund and M. A. Rubin, "Dynamics of Dimensional Reduction," Phys. Lett. B97 (1980) 233-235.

[26] E. Silverstein, "TASI / PiTP / ISS lectures on moduli and microphysics," hep-th/0405068.

[27] S. Kachru et. al., "Towards inflation in string theory," JCAP 0310 (2003) 013, hep-th/0308055.

[28] I. C. G. Campbell, "The Stability of ten-dimensional Kaluza-Klein Supergravity," Phys. Rev. D31 (1985) 1911.

[29] L. Susskind and E. Witten, "The holographic bound in anti-de Sitter space," hep-th/9805114.

[30] O. DeWolfe, S. Kachru, and H. L. Verlinde, "The giant inflaton," JHEP 05 (2004) 017, hep-th/0403123

[31] A. Sen, "F-theory and Orientifolds," Nucl. Phys. B475 (1996) 562-578, hep-th/9605150.

[32] A. R. Frey and J. Polchinski, "N = 3 warped compactifications," Phys. Rev. D65 (2002) 126009, hep-th/0201029.

[33] S. Kachru, M. B. Schulz, and S. Trivedi, "Moduli stabilization from fluxes in a simple IIB orientifold," JHEP 10 (2003) 007, hep-th/0201028.

[34] P. Breitenlohner and D. Z. Freedman, "Stability in Gauged Extended Supergravity," Ann. Phys. 144 (1982) 249.

[35] M. J. Duff, B. E. W. Nilsson, and C. N. Pope, "THE Criterion for Vacuum Stability in Kaluza-Klein Supergravity," Phys. Lett. B139 (1984) 154. 
[36] P. van Nieuwenhuizen, "The Complete Mass Spectrum of d = 11 Supergravity Compactified on S(4) and a General Mass Formula for Arbitrary Cosets M(4)," Class. Quant. Grav. 2 (1985) 1.

[37] B. Biran, A. Casher, F. Englert, M. Rooman, and P. Spindel, "The Fluctuating Seven Sphere in Eleven-Dimensional Supergravity," Phys. Lett. B134 (1984) 179.

[38] O. DeWolfe, D. Z. Freedman, S. S. Gubser, G. T. Horowitz, and I. Mitra, "Stability of AdS(p) x M(q) compactifications without supersymmetry," Phys. Rev. D65 (2002) 064033, hep-th/0105047.

[39] S. Gukov, S. Kachru, X. Liu, and L. McAllister, "Heterotic moduli stabilization with fractional Chern-Simons invariants," Phys. Rev. D69 (2004) 086008, hep-th/0310159.

[40] T. Shiromizu, D. Ida, H. Ochiai, and T. Torii, "Stability of AdS(p) x S(n) x S(q-n) compactifications," Phys. Rev. D64 (2001) 084025, hep-th/0106265.

[41] J. Polchinski, "String theory. Vol. 2: Superstring theory and beyond,". Cambridge, UK: Univ. Pr. (1998) 531 p.

[42] S. B. Giddings, S. Kachru, and J. Polchinski, "Hierarchies from fluxes in string compactifications," Phys. Rev. D66 (2002) 106006, hep-th/0105097.

[43] M. Grana and J. Polchinski, "Supersymmetric three-form flux perturbations on AdS(5)," Phys. Rev. D63 (2001) 026001, hep-th/0009211.

[44] S. S. Gubser, "Supersymmetry and F-theory realization of the deformed conifold with three-form flux," hep-th/0010010.

[45] E. Silverstein, "AdS and dS entropy from string junctions or the function of junction conjunctions," hep-th/0308175

[46] M. J. Duff, B. E. W. Nilsson, and C. N. Pope, "Kaluza-Klein Supergravity," Phys. Rept. 130 (1986) 1-142. 